\title{
Shot peen forming pattern optimization to achieve complex target shapes: the inverse problem
}

\author{
Hong Yang Miao, Martin Lévesque, Frédérick P. Gosselin \\ Laboratory for Multiscale Mechanics (LMㄴ), Aluminium Research Centre - REGAL, \\ Department of Mechanical Engineering, Ecole Polytechnique Montréal, Montréal, Québec, \\ Canada.
}

\begin{abstract}
The inverse problem of determining how to shot peen a plate such that it deforms into the desired target shape is a challenge in the peen forming industry. While peening thick plates uniformly on one side results in a spherical shape, with the same curvature in all directions, complex peening patterns are required to form other shapes, such as cylinders and saddles found on fuselages and wing skin panels. In this study, we present an optimization procedure to automatically compute shot peening patterns. This procedure relies on an idealized model of the peen forming process, where the effect of the treatment is modeled by in-plane expansion of the peened areas, and on an off-the-shelf optimization algorithm. For validation purposes, we peen formed three $305 \times 305 \times 4.9 \mathrm{~mm}$

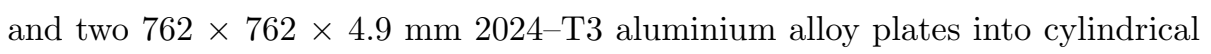
and saddle shapes using the same peening treatment. The obtained shapes qualitatively match simulations. For $305 \times 305 \times 4.9 \mathrm{~mm}$ plates, the relative differences had the same distribution and were of the same order of magnitude as initial out-of-plane deviations measured on the as-received plates.
\end{abstract}

Keywords: Shot peening, Peen forming, Optimization, Eigenstrains, Finite element method, Residual stresses, 2024-T3 aluminium alloy

Email address: frederick.gosselin@polymtl.ca (Frédérick P. Gosselin)

URL: https://www.polymtl.ca/lm2 (Frédérick P. Gosselin)

Preprint submitted to engrXiv

June 23, 2021 


\section{Introduction}

During the shot peen forming process, small shot propelled at several tens of meters per second are used to plastically deform the surface layer of a thin part, causing it to deform, as illustrated in Figure 1. Miao et al. (2010) experimentally demonstrated that uniformly shot peening a plate of uniform thickness with the same intensity and coverage deforms it into a spherical shape, as illustrated in Figure 1(e). However, most industrial applications demand more complex geometries such as combination of cylindrical or saddle shapes. One way to do so is to vary the intensity and/or the density of impacts over the peened surface. Another technique, called stress peen forming, consists in elastically pre-straining the part in specific directions. The process results in larger plastic strains, hence larger curvatures, in the direction in which the part is stretched. Hornauer and Kohler (1990) peen formed tank bulkheads of the ARIANE 5 launcher with a tolerance of $1.5 \mathrm{~mm}$ from a nominal radius of 3000 $\mathrm{mm}$ using the stress peen forming technique. When compared to conventional peen forming at room temperature, warm peen forming at a temperature below the aging temperature of the material increases the bending capability of the treated panel to achieve further curvature. Ramati et al. (1999) combined the

(a)
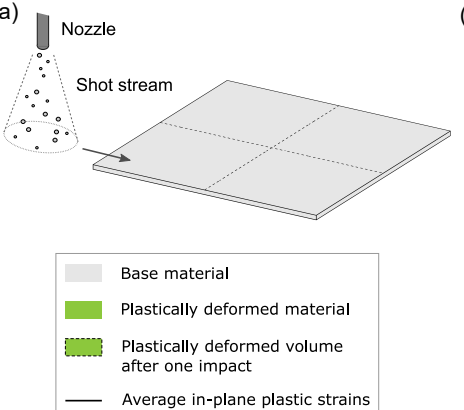

(b)

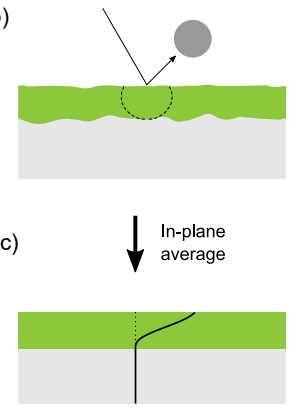

(d)

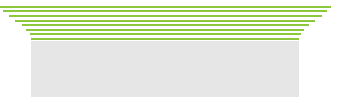

(e)

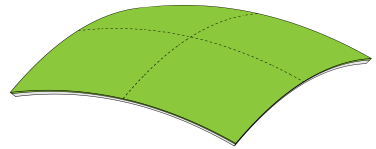

Figure 1: Schematic illustration of the shot peen forming process. (a) A high speed shot stream bombards a metallic surface. (b) Shot impacts on the surface create a plastically deformed layer. (c) Average in-plane plastic strain distribution through the thickness of the surface layer. (d) The plastically deformed layer stretches the surface of the material. (e) Uniformly shot peening the surface induces a spherical shape from an initially flat plate. 
conventional peen forming, the stress peen forming and the warm peen forming techniques to form complex industrial parts such as a wing skin panel with different curvatures, chordwise and spanwise. However, in the previously cited studies, the peen forming sequences were developed by trial and error attempts, which are time consuming and which severely restrict the number of parts that are economically viable to peen form.

Most existing shot peen forming studies seek to numerically simulate the deflections of a given part under a specified shot peening treatment, which are called the direct problem. Wang et al. (2006) developed a finite element model to simulate the deflection of unconstrained Aluminium 5251-H22 specimens uniformly peen formed with S660 media. Three different equivalent thermal loads were applied to represent the equivalent plastic strain after peen forming with different peening pressures. However, the treated specimens were limited to dimensions of $76 \times 19 \times 3 \mathrm{~mm}$ and a treated area of $19 \times 19 \mathrm{~mm}$. Faucheux et al. (2018) simulated the peen forming process on larger panels having dimensions of $1000 \times 1000 \mathrm{~mm}$ and thickness of 5,10 and $15 \mathrm{~mm}$. Eigenstrain profiles after shot peening were derived from experimentally measured residual stress profiles and were reconstructed by two parameters: depth of idealized eigenstrain profile $h_{e q}$ and its amplitude $\epsilon_{e q}^{*}$ to simplify the finite element analysis. The simulations successfully explained the elastic instability which causes the transition from spherical to cylindrical shapes as the panel thickness decreased 40 from $15 \mathrm{~mm}$ to $5 \mathrm{~mm}$.

In practice, however, it is the inverse problem that process engineers are faced with-i.e., determining the optimal peening parameters, such as intensity, coverage, peening trajectory, and peening pattern, etc., to achieve a prescribed target shape. To solve this inverse problem, it is necessary to develop a numerical optimization model that minimizes the deviation of the computed deformed shape from the desired shape subject to certain shot peening treatments.

Few investigations on process optimization procedures were reported in the shot peening literature and most of them were only validated on relatively small plates. VanLuchene and Cramer (1996) developed an optimization model to de- 
termine the optimal peening intensity pattern required to produce an arbitrary aerodynamic contour. Their approach was based on a finite element shell model of the part to be formed and used the amplitude of stretching and bending loads in each element as optimization variables. Nevertheless, although it was applied to form actual wing skins, neither typical patterns nor experimental re55 sults were reported. Kopp and Schulz (2002) applied their previous established optimization tool to establish a direct relationship between the objective shapes and the process parameters to form three-dimensional contours by peening both sides of a plate. Using the optimized peening parameters, seats with wave or saddle contours were produced as a demonstrator. However, little detail about the optimization was provided in their report. Wang et al. (2008) developed an optimization method to minimize the difference between the target shape and peen formed shape based on a simple non-linear relationship assumption between the deformed arc height and the peening time. Since the model was defined as a one-dimensional problem, the model was validated on a cylindrical target shape on a small aluminium 5251-H22 sheet with dimensions of $76 \times 76 \times$ $3 \mathrm{~mm}$. Siguerdidjane et al. (2020) developed a method to find the peen forming patterns based on a neural network. The authors trained a neural network with thousands of finite element simulations of the direct problem. Once the costly off-line task of generating the simulation data and training was done, the neural network could predict in a fraction of a second the shot peening pattern which would yield the wanted shape. However, this approach has not yet been validated and the off-line task of generating thousands of simulation is costly.

Inverse methods have been developed for laser peen forming applications. Thomson and Pridham (1997) presented an application of a closed-loop con75 trol system for the laser peen forming process. When compared to a predictive approach that uses empirical and theoretical method to determine the process parameters, the feedback approach monitors the deformation of the plate with the attached sensors, compares the real time resulting deflections with the target shape, and finally adjusts the process parameters. Experimental tests showed that the proposed closed-loop control approach produced better results 
than the predictive approach. However, the system produced some variabilities, especially when the deflections were close to the target shape. A maximum tolerance of $0.4 \mathrm{~mm}$ above the target deflection were presented in a series of 18 forming tests with target deflections between 1 to $6 \mathrm{~mm}$. Therefore, further improvements were required to reach a greater degree of precision to meet industrial applications requirements. Recently, Luo et al. (2020) developed a partial differential equation constrained optimization method, combined with a distributed eigen-moment, to compute the laser shot peening process parameters yielding a target saddle shape. The process was validated by forming a $272 \times$ $272 \times 4 \mathrm{~mm}$ 2024-T351 aluminium alloy plate into a saddle shape. Experimental results qualitatively matched the desired shape with a maximum difference of $0.35 \mathrm{~mm}$ over a maximum deformation of $4.5 \mathrm{~mm}$.

Although the literature on optimizing peening patterns is scarce, many robust optimization procedures were proposed to address closely related problems encountered in other fields of engineering. For example, Koconis et al. (1994) developed an analytical model to determine the required voltages to achieve a specified target shape on fiber-enforced composite beams, plates and shells with embedded piezoelectric actuators. Paradies and Hertwig (1999) presented an adaptive composite mirror with integrated piezoelectric actuators to compensate their out-of plane displacements due to their self-weight. Irschik (2002) reviewed studies for the shape control of smart structures with piezoelectric control actuation. There are striking similarities between peen forming and shape control of thin structures with piezoelectric actuators: in both cases, a thin multilayered structure deforms in response to the incompatible expansion of its outer layers and the objective is to find an optimal loading for the plate to achieve the desired target shapes. Pajot et al. (2006) presented one example of such a system where a patterned gold film was deposited onto a polysilicon substrate such that the difference of thermal expansion between the two layers caused the structure to deform into a prescribed target shape when cooled down. The optimal patterns were computed with a procedure derived from topology optimization - similar to the solid isotropic material with penalization (SIMP) 
method described in Bendsoe and Sigmund (2013) - where a finite element model

of the polysilicon-gold substrate was used to compute deformed shapes and a gradient-based optimizer was used to generate patterns.

In this paper, we propose a procedure to automatically compute shot peening patterns to achieve the pre-defined shapes. Our procedure is adapted from the multilayered structure presented in Pajot et al. (2006), the SIMP method of Bendsoe and Sigmund (2013) and combined with the eigenstrain approach for modeling the effect of shot peening by Faucheux et al. (2018). Section2 describes a numerical procedure to automatically compute peening patterns required to form flat plates into desired target shapes. Section 3 presents materials and methods. Section 4 presents the simulated optimized shot peening patterns to achieve cylindrical and saddle shapes and the validations with experimental results. Finally, Sections 5 and 6 discuss key results and conclude this work.

\section{Overview of the numerical optimization procedure}

\subsection{Computing deformed shapes for specified peening patterns: the direct prob- lem}

To optimize peening patterns, we first need a model to predict how a shot peened plate deforms. Here, we used an idealized model similar to that described

130 in Faucheux et al. (2018), where the plates are meshed with thin-shell elements and composite section properties — with a passive inner layer and two active outer layers that can expand - to mimic the effect of the peening treatment. The amount of expansion was controlled by adjusting the value of the in-plane thermal expansion coefficient in each element and the whole model were loaded by applying a unit temperature increment.

\subsubsection{Finite element model}

All simulations were done with an in-house finite element code using the discrete Kirchhoff triangle DKT18 thin-plate elements described in Batoz and Dhatt (1992). As illustrated in Figure 2(a), the plates were discretized with 
(a)

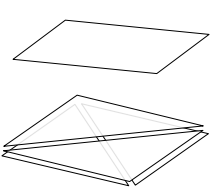

(c)

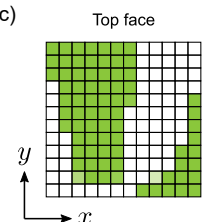

(b)
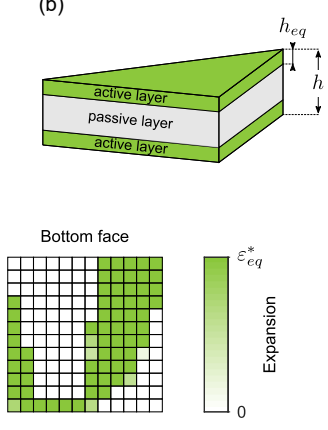

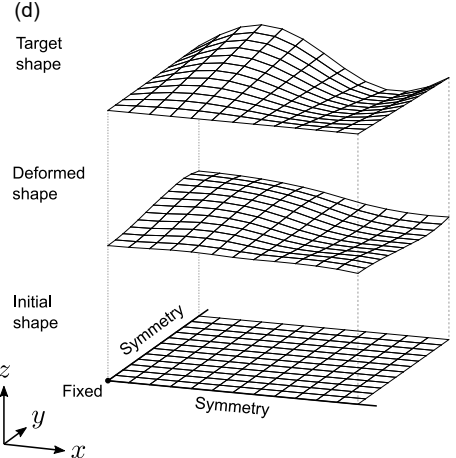

Figure 2: Schematic illustration of the optimization procedure that identifies the peening pattern to form a thin flat plate into a 3D surface. (a) The plate is meshed with rectangular patches of 4 superimposed DKT18 elements. (b) Peening-induced loads are imposed as an inplane expansion in the top and bottom surface layers with thickness of $h_{e q}$, which are active layers. The middle of the plate is considered as a passive layer. (c) The peening patterns during the optimization process for the top and bottom faces. The expansion on each face of each patch is varied between 0 and an upper bound to represent unpeened, partially peened, and totally peened areas. (d) A model of an initially flat plate deforms as loads representative of the peening process are applied to it. The optimization procedure attempts to minimize the z-coordinate error between the deformed shape and a prescribed target shape. 

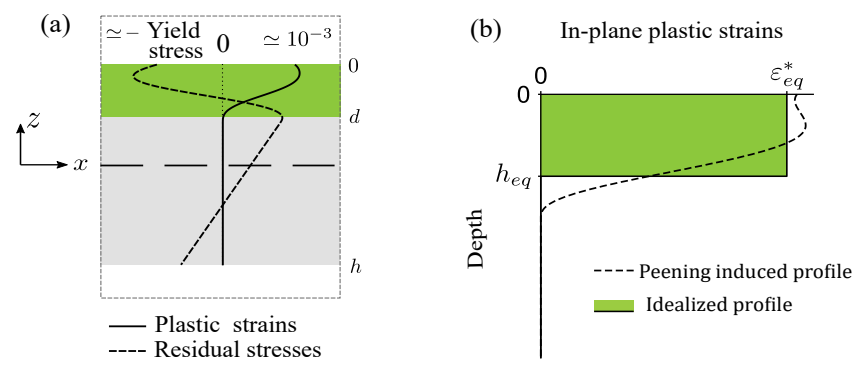

Figure 3: Surface eigen strain for the calculation of deflection of the plate. (a) Plastic strains and a balanced compressive residual stress profile are produced in the depth $h$ under the surface of the plate. (b) An idealized 'step' profile with an equivalent depth $h_{e q}$ and in-plane expansion $\varepsilon_{e q}^{*}$ are defined to impose the peening induced plastic strain profile in the finite element simulation model that yields the same stretching and bending as the real plastic deformation profile.

rectangular patches and each patch was meshed with four triangular DKT18 elements. We model the peening pattern by controlling the expansion of the three layers. The upper and lower layers of all elements in a patch are linked to a single optimization variable $\boldsymbol{\chi}$, a vector of peening intensities for all patches $i=1, \ldots, \mathrm{n}$, where $\chi_{i}=1$ corresponds to a fully peened area and $\chi_{i}=0$ corresponds to an unpeened area. The amplitude of the expansion profile input in the upper and lower layers of those elements was equal to the original input eigenstrain profile, introduced in Section 2.1.2 multiplied by $\chi_{i}$.

Only a quarter of the plate was simulated using two symmetry boundary condition. All translations and rotations of the node located at $(0,0,0)$ were set to zero to prevent rigid body motions.

For nonlinear simulations, we implemented the small strain corotational formulation presented in Caselli and Bisegna (2013) using the same DKT18 element as a core element - similarly to what was done in Pajot et al. (2006). Corotational formulations are appropriate for the problem at hand because they make the computation of gradients with respect to the magnitude of follower loads (which are the optimization variables used here) much easier than with total or updated Lagrangian formulations (Pajot and Maute, 2006). 


\subsubsection{Loads}

We reconstructed the eigenstrain profiles for the studied material and the peening treatment from the measured residual stress using the inverse procedure described in Faucheux et al. (2018). However, because we are only interested in the deformed shape of the plates, it is simpler to input an idealized expansion profile that induces the same axial load and bending moment. When thin-shell elements are used, profiles having the same in-plane and bending resulting loads yield identical deformed shapes. Therefore, it is not necessary to input the full expansion profile in the model since loads are integrated over the thickness of the element. In what follows, Faucheux et al. (2018) used idealized 'step' profiles, as shown in Figure 3(b), which are fully characterized by an equivalent depth $h_{e q}$ and an equivalent in-plane expansion $\varepsilon_{e q}^{*}$. The active upper and lower layers of thickness $h_{e q}$ could expand, while, the central passive layer could not expand.

The depth $h_{e q}$ and the amplitude $\varepsilon_{e q}^{*}$ of the equivalent eigenstrain profiles used for simulations were obtained by requiring that they yield the same resulting eigenstrains $\alpha$ and the same first eigenstrain moment $\beta$ as profiles computed from the experimentally measured residual stress profile. This condition can be written as

$$
h_{e q}=2 \beta / \alpha, \quad \varepsilon_{e q}^{*}=\alpha^{2} / 2 \beta .
$$

\subsection{Computing peening patterns for specified target shapes: the inverse problem}

The goal of the optimization procedure is to find a combination of optimization variables that best-fits a given target shape, as shown in Figure 2(d). We introduce the vector of peening intensities $\chi$, and we seek to optimize:

$$
\varepsilon^{*}(h)= \begin{cases}\varepsilon_{e q}^{*} \chi & \text { if } h \leq h_{e q}, \\ 0 & \text { if } h>h_{e q},\end{cases}
$$

The optimization problem is defined as,

$$
\begin{array}{lll}
\text { minimize } & \left\|\boldsymbol{z}(x, y, \boldsymbol{\chi})-\boldsymbol{z}_{\text {target }}(x, y)\right\|_{2}^{2} & \\
\text { subject to } & 0 \leq \chi_{i} \leq 1, & \\
& \boldsymbol{A}^{T} \boldsymbol{\chi} \leq A_{\max }, &
\end{array}
$$


where $\boldsymbol{z}$ is the vector of current nodal coordinates in the $z$-direction, $\boldsymbol{z}_{\text {target }}$ is a vector that contains coordinates of the target shape in the $z$-direction evaluated at the location of the nodes, $\chi$ is the vector of peening intensities for all patches, introduced in Section 2.1.1, $\boldsymbol{A}$ is a vector that contains the area of the patches associated with each optimization variable, and $A_{\max }$ is a user-defined upper bound that limits the total area that can be peened. It is implicitly understood that deformed nodal coordinates are computed with the finite element model of the structure and that the computed solution satisfies equilibrium, compatibility, the constitutive law, and boundary conditions. The constraint $\boldsymbol{A}^{T} \chi \leq A_{\max }$ was used to steer the solution towards easy-to-peen [0-1] patterns, as progressively decreasing the value of $A_{\max }$ forces the optimization algorithm to allocate peened areas only where this has the most impact on the shape of the plate. This penalizes pattern with areas with intermediate values of $\chi$ or checkerboard.

Matlab's (R2018a) fmincon interior point algorithm was used for the optimization with the default termination criteria. The cost function and second constraint were scaled by $\max \left(\boldsymbol{z}_{\text {target }}\right) / 100$ and $A_{\max }$, respectively. In all the examples presented hereafter, simulations were systematically initialized from several initial random guesses of $\chi$ as multiple optimal solutions could exist. In all cases, the procedure converged towards the same solution irrespective of the initialization.

\section{Experimental tests and Materials}

\subsection{Peen forming process}

Initially flat plates were laid horizontally and shot peened in the Canablast compressed-air cabinet shown in Figure 4. The shot peening machine is equipped with a 6 -axis robotic arm for accurate positioning of the nozzle (M-20iA supplied by Fanuc), with a particle velocity sensor to measure the average shot velocity (Shotmeter G3 supplied by Progressive Technologies), and with a GoPro digital camera to record the tests. The plates were secured with double 

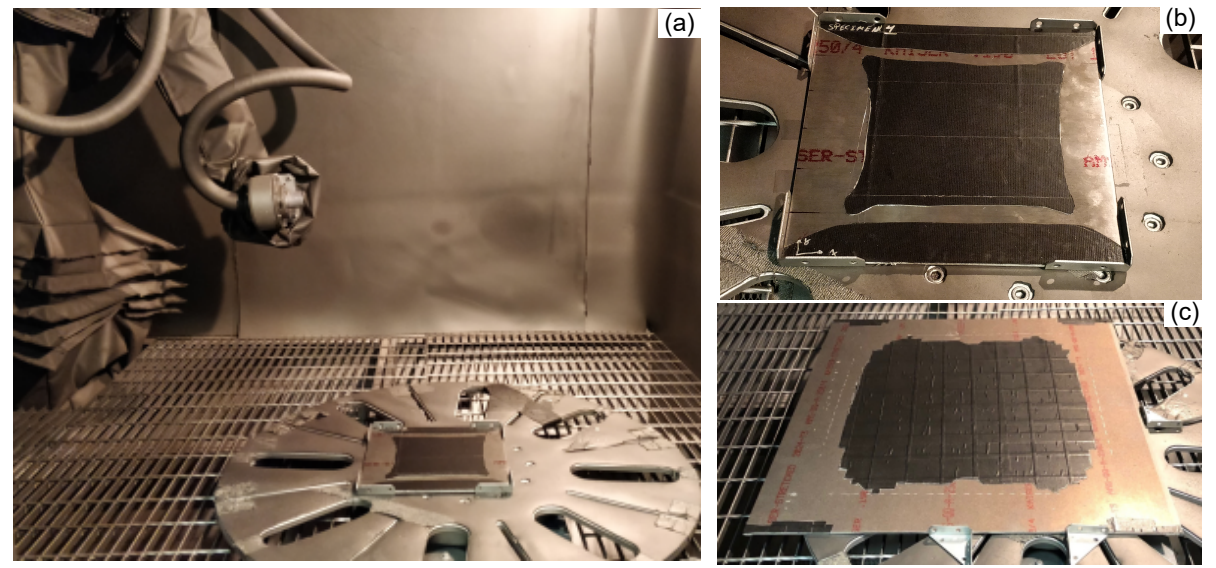

Figure 4: Shot peening setup and treated plates with masks for certain peening patterns. (a) Compressed air peening cabinet used for peen forming tests. During the peen forming tests, the plate was free to deform. (b) The bottom face of plate P1-S-Sphere was masked by tapes according to a simulated peening pattern to achieve a cylindrical shape. The four corners of the plate was fixed to prevent the movement of the plate (c) The bottom face of plate P5-L-Saddle was masked by tapes according to a simulated peening pattern to achieve a saddle shape. Two sides of the plate were fixed with holders to prevent its movement.

sided tape on their bottom surfaces and with holders on their corners or sides to prevent their movement during shot peening.

The plates were peened with SCCW28 media having a hardness of 55-62 HRC and a nominal diameter of $0.71 \mathrm{~mm}$, an air pressure of $0.172 \mathrm{MPa}$, a mass flow of $0.12 \mathrm{~kg} / \mathrm{s}$, a shot speed of $41 \mathrm{~m} / \mathrm{s}$, and the nozzle was at a stand-off distance of $41 \mathrm{~mm}$. An Almen intensity of $0.3 \mathrm{mmA}(12 \mathrm{~A})$, as defined in SAE standard SAEJ443, and a coverage of $100 \%$, as defined in SAE standard SAE standard J2277, were applied during all the peen forming tests AMS Standard 2430). According to the simulated [0-1] peening pattern, 1 represents the surface peened with $100 \%$ coverage, 0 represents the surface without peening and was masked with duct tape during the peening process, as shown in Figure 4 (c).

\subsection{Peen forming residual stress and calculation of eigenstrain profile}

A 2024-T3 aluminium alloy plate having dimensions of $1016 \times 254 \times 4.9 \mathrm{~mm}$ was shot peened at an Almen intensity of $0.3 \mathrm{mmA}(12 \mathrm{~A})$ and $100 \%$ coverage 
using SCCW28 media. A coupon having dimensions of $254 \times 254 \mathrm{~mm}$ was

removed from the center of peen formed plate with a lubricated jigsaw and was sent to Hill-Engineering, Rancho Cordova, California to measure the residual stresses with a hole drilling strain gage method as per ASTM standard E837-08 Residual stresses were measured at the center of the coupon, far from the edges, to minimize the effect of cutting-induced plastic deformations and heating on measurement. The holes were $2 \mathrm{~mm}$ in diameter and were drilled in $0.051 \mathrm{~mm}$ increments to a final depth of $1.020 \mathrm{~mm}$.

Figure 5(a) presents the measured residual stress profile along the plate's rolling (L) and transverse $(\mathrm{T})$ directions. Peen forming was performed using SCCW28 media with an Almen intensity of $0.3 \mathrm{mmA}(12 \mathrm{~A})$ and a coverage of $100 \%$. Figure 5(b) reports the calculated peening induced eigenstrains using the method introduced in 2.1. Using the average eigenstrain profiles of the $\mathrm{L}$ and $\mathrm{T}$ directions in Figure $5(\mathrm{~b}), h_{e q}=0.31 \mathrm{~mm}$ and $\varepsilon_{e q}^{*}=4.1 \times 10^{-3}$ were computed from Equation 1. The computed eigenstrain profiles were then modelled as anelastic in-plane expansions of the surface in peened areas.

\subsection{Materials samples for the validation of the optimized peening pattern}

Three 2024-T3 aluminium alloy plates having dimensions of $305 \times 305 \times 4.9 \mathrm{~mm}$ and two plates having dimensions of $762 \times 762 \times 4.9 \mathrm{~mm}$ were peened according the optimized peening patterns computed to form them into the target shapes. The dimensions of the smaller plates were chosen such that the peening treatment induced small out-of-plane displacements compared to the thickness of the plate, and that they could be predicted with a linear model. On the other hand, the dimensions of the larger plates were chosen such that out-of-planes deflections were larger than the thickness of the plate. Modeling the response of these plates therefore required a geometrically nonlinear model.

The target shape of the plate having dimensions of $305 \times 305 \times 4.9 \mathrm{~mm}$ (P1-S-Cylinder) was a cylinder. The two plates having dimensions of $305 \times$ $305 \times 4.9 \mathrm{~mm}$ (P2-S-Saddle and P3-S-Saddle) and the two larger plates having dimensions of $762 \times 762 \times 4.9 \mathrm{~mm}$ (P4-L-Saddle and P5-L-Saddle) were to be 
(a)

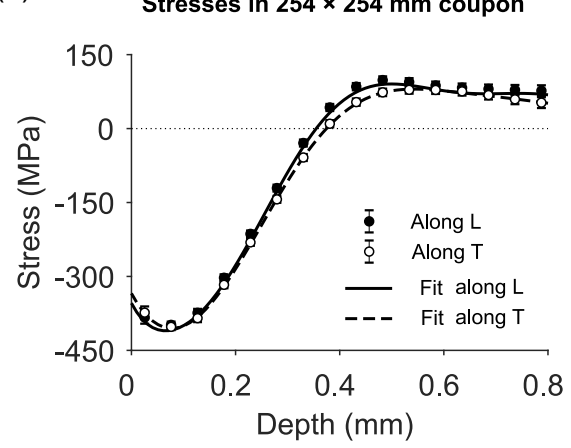

(b)

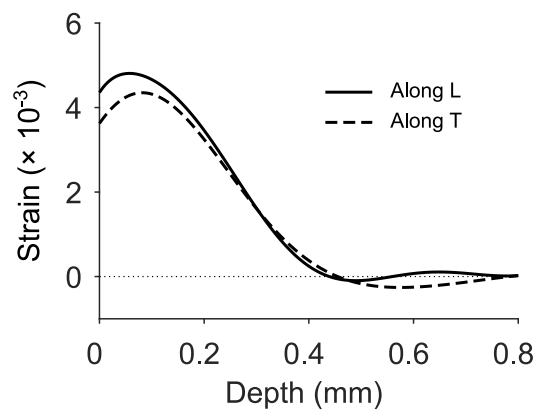

Figure 5: Residual stress and reconstructed eigenstrain profiles in peen formed 2024-T3 aluminium alloy specimen along rolling $(\mathrm{L})$ and transverse $(\mathrm{T})$ directions. Peen forming was performed using SCCW28 media with an Almen intensity of $0.3 \mathrm{mmA}(12 \mathrm{~A})$ and a coverage of $100 \%$. (a) Residual stresses measured with the hole drilling method at the center of the coupon having dimensions of $254 \times 254 \mathrm{~mm}$, which was removed from the center of a peen formed specimen having dimension of $1016(\mathrm{~L}) \times 254(\mathrm{~T}) \times 4.9 \mathrm{~mm}$. The fitting curvesshown only to guide the eye - are polynomial fits. (b) Peening-induced eigenstrains computed from the residual stress profile using the inverse method introduced in (Faucheux et al., 2018).

formed as saddles and required respectively a linear and a nonlinear code. The target cylindrical and saddle shapes were defined as

$$
z(x, y)=\frac{1}{2}\left(\kappa_{x} x^{2}+\kappa_{y} y^{2}\right)
$$

where $\kappa_{x}$ and $\kappa_{y}$ are the curvatures of the target shape in the $x$ - and $y$-directions, respectively. The coordinate $(0,0,0)$ was defined at the middle of the plate. Table 1 lists the information of the five plates considered for the optimization procedure validation. A Young's modulus of $71.5 \mathrm{GPa}$ and a Poisson ratio of 0.33, taken from the MMPDS-08 handbook, were used for all simulations.

\subsection{D scans and curvature measurements}

All five peen formed plates were scanned with a coordinate measuring machine (Mitutoyo, Crysta-Apex 163011) equipped with a REVO $^{\circledR} 5$-axis measurement system. Measurements were taken every $4 \mathrm{~mm}$ in continuous scanning mode along several lines parallel to the $x$ and $y$ directions of the plate. 
Table 1: The plates dimensions, target shapes, curvatures of the target shapes, the maximum deflection of the target shapes $z(x, y)_{\text {tar }}$ and the code types used for the optimization procedure.

\begin{tabular}{lllllll}
\hline Plate name & $\begin{array}{l}\text { Plate dimensions } \\
(\mathrm{mm} \times \mathrm{mm} \times \mathrm{mm})\end{array}$ & Target shape & $\begin{array}{l}\kappa_{x} \\
\left(\times 10^{-4} \mathrm{~mm}^{-1}\right)\end{array}$ & $\begin{array}{l}\kappa_{y} \\
\left(\times 10^{-4} \mathrm{~mm}-1\right)\end{array}$ & $\begin{array}{l}\text { Maximum } z(x, y) \\
\text { tar } \\
(\mathrm{mm})\end{array}$ & $\begin{array}{l}\text { Linear/Nonlinear } \\
\text { Code }\end{array}$ \\
\hline P1-S-Cylinder & $305 \times 305 \times 4.9$ & Cylinder & -1.50 & 0.00 & 1.74 & Linear \\
P2-S-Saddle & $305 \times 305 \times 4.9$ & Saddle & -1.08 & 1.08 & 2.51 & Linear \\
P3-S-Saddle & $305 \times 305 \times 4.9$ & Saddle & -1.08 & 1.08 & 2.51 & Linear \\
P4-L-Saddle & $762 \times 762 \times 4.9$ & Saddle & -1.00 & 1.00 & 14.52 & Linear \\
P5-L-Saddle & $762 \times 762 \times 4.9$ & Saddle & -1.00 & 1.00 & 14.52 & Nonlinear \\
\hline
\end{tabular}

3.5. Evaluation of the differences and relative differences between target shapes, simulation and experiments

Several differences and relative differences were defined to compare the resulting shapes to the target shapes, namely:

$$
\begin{array}{cc}
\mathrm{D}_{s-t}=z(x, y)_{s i m}-z(x, y)_{t a r}, \quad & \mathrm{RD}_{s-t}=\frac{\left|\mathrm{D}_{s-t}\right|}{z(x, y)_{\text {tar }}} \times 100 \%, \\
\mathrm{D}_{i-t}=z(x, y)_{\text {ini }}-z(x, y)_{t a r}, & \mathrm{RD}_{i-t}=\frac{\left|\mathrm{D}_{i-t}\right|}{z(x, y)_{\text {tar }}} \times 100 \%, \\
\mathrm{D}_{e-s}=z(x, y)_{\text {exp }}-z(x, y)_{s i m}, \quad \mathrm{RD}_{e-s}=\frac{\left|\mathrm{D}_{e-s}\right|}{z(x, y)_{\text {sim }}} \times 100 \%, \\
\mathrm{D}_{e-o}=z(x, y)_{\text {exp }}-z(x, y)_{t a r}, \quad \mathrm{RD}_{e-t}=\frac{\left|\mathrm{D}_{e-t}\right|}{z(x, y)_{\text {tar }}} \times 100 \%,
\end{array}
$$

where $z(x, y)_{t a r}, z(x, y)_{s i m}, z(x, y)_{i n i}$, and $z(x, y)_{\exp }$ are the target shape, the simulated optimal shape, the as-received initial shape, and the experimental shape of the plate respectively. $\mathrm{D}_{s-t}, \mathrm{RD}_{s-t}, \mathrm{D}_{i-t}, \mathrm{RD}_{i-t}, \mathrm{D}_{e-s}, \mathrm{RD}_{e-s}$, and $\mathrm{D}_{e-t}, \mathrm{RD}_{e-t}$ are the absolute differences and relative differences between simulated optimal and target shapes, initial and target shapes, experimentally measured and simulated optimal shapes, and experimentally measured and target shapes, respectively. 


\section{Results}

260

4.1.1. Comparison between the target shape and that predicted from the optimized peening pattern

Figure 6 shows the target cylindrical (a) and saddle (e) shapes, the simulated optimal [0-1] peening patterns (b, f), the simulated optimization shapes (c, shape $\mathrm{D}_{s-t}(\mathrm{~d}, \mathrm{~h})$ for cylindrical (P1-S-Cylinder) and saddle (P2-S-Saddle and P3-S-Saddle) target shapes, respectively. The green color area on the plate represents the peening region and the white area on the plate represents the unpeened region in the experiments. The light green area in Figure 6 (b), where $\chi=0.27$ or shot peening coverage of $27 \%$, represents a 'partially peened areas' area resulting from the optimization code. To achieve the pattern with a single treatment, the area with $\chi<0.4$ were masked during the experiments.

In Figure 6 $(\mathrm{d}, \mathrm{h})$, the difference between the simulated optimal shapes and the target shapes $\mathrm{D}_{s-t}$ is smaller than $0.08 \mathrm{~mm}$ for both the cylindrical and the

differences $\mathrm{D}_{s-t}$ of $\pm 0.22 \mathrm{~mm}$ and $\pm 0.4 \mathrm{~mm}$ were concentrated on the four edges of the plate for both the cylindrical and the saddle shapes.

\subsubsection{Comparison between experimentally peened plates and simulated optimal shapes}

Figure 7 presents the three-dimensional scans of the four plates peened with the simulated peening patterns of Figure 6 and their differences with respect to the simulated target shapes $\mathrm{D}_{e-s}$. The three-dimensional scans revealed that all the plates qualitatively exhibited the desired target shape. It should be noted that, for the purpose of peening pattern optimization, the plates were considered as perfectly flat. However, Figs. 7 (b, f, j) show that the plates were not perfectly flat prior to peening. Plate P1-S-Cylinder had an initial deflection $\mathrm{D}_{\text {ini }}$ of $0.23 \mathrm{~mm}$ and the maximum difference between peen formed shape and 
P1-S-Cylinder

(a)

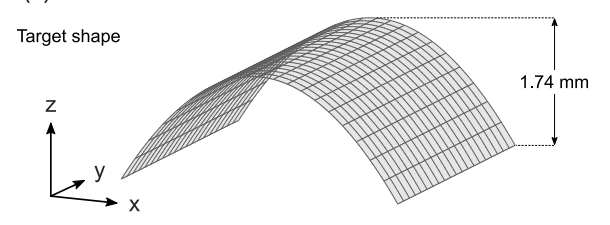

(b) Peening pattern Top face
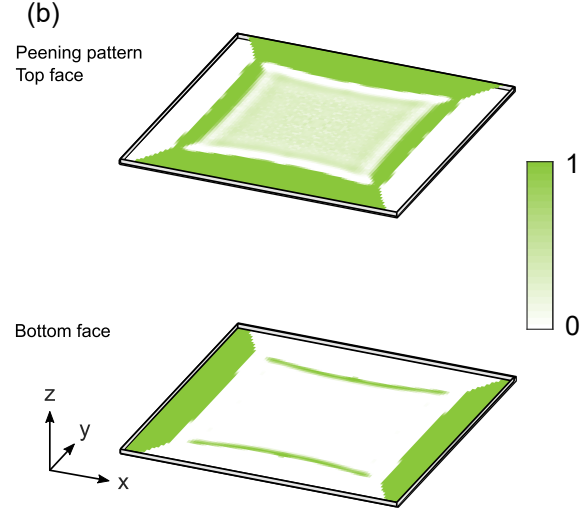

(c) Deformed shape
(Finite elements)

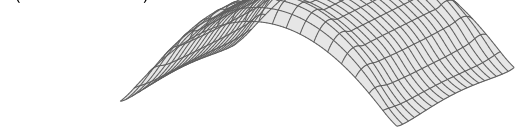

(d) $D_{s-t}$ (in $\mathrm{mm}$ )

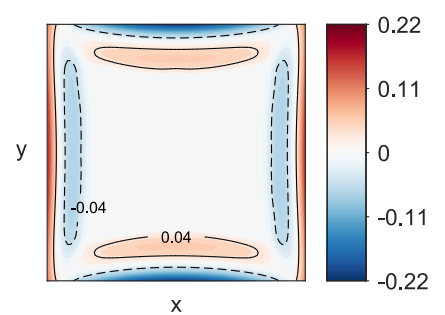

P2-S-Saddle, P3-S-Saddle

(e)

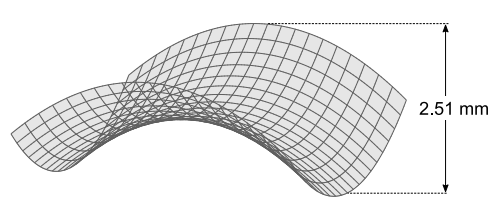

(f)

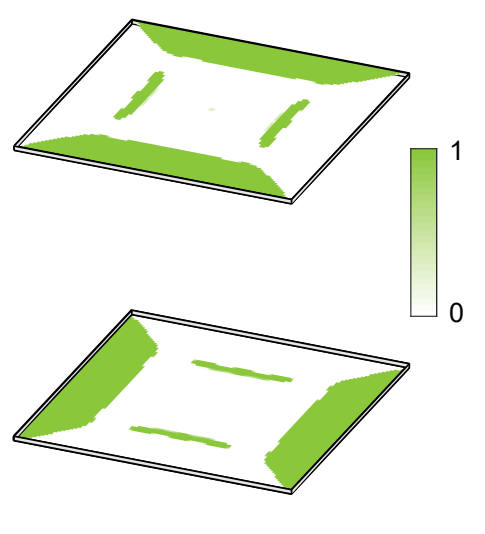

(g)

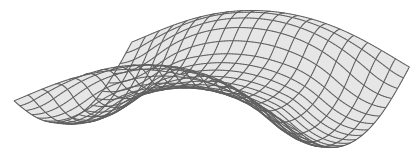

(h)

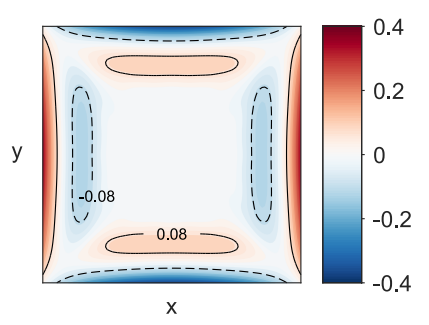

Figure 6: Peening patterns to achieve a cylindrical shape for plate P1-S-Cylinder and saddle shapes for plates P2-S-Saddle and P3-S-Saddle. (a) Target cylindrical shape having a target deflection $\mathrm{D}_{\text {tar }}$ of $1.74 \mathrm{~mm}$ for plate P1-S-Cylinder. (b) Optimized peening patterns on the top and bottom faces of the plate to achieve a cylindrical shape. The light green area in the middle of the plate, where $\chi=0.27$, represents a surface with $27 \%$ coverage. (c) Simulated optimal cylindrical shape $z(x, y)_{\text {sim }}$. (d) Difference between the simulated optimization shape and the target cylindrical shape $\mathrm{D}_{s-t}$ with a maximum value of $\pm 0.22 \mathrm{~mm}$. (e) Target saddle shape having a target deflection $\mathrm{D}_{\text {tar }}$ of $2.51 \mathrm{~mm}$ for plates P2-S-Saddle and P3-S-Saddle. (f) Optimized peening patterns on top and bottom faces of the plate to achieve a saddle shape. (g) Simulated optimal saddle shape $z(166)$ sim. (h) Difference between the simulated optimization shape and the target saddle shape $\mathrm{D}_{s-t}$ with a maximum value of $\pm 0.40 \mathrm{~mm}$. 


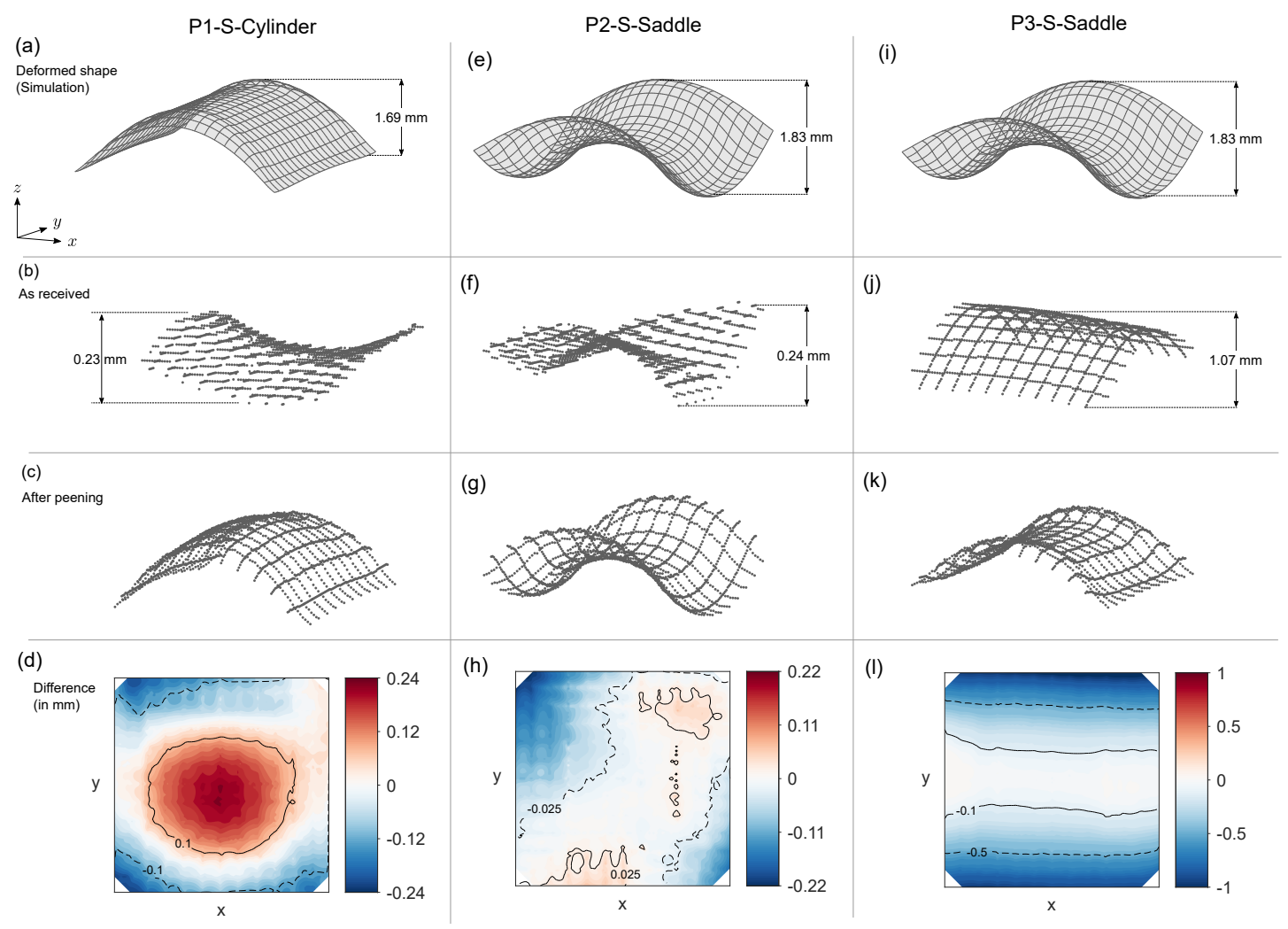

Figure 7: Comparison between the peen formed shapes and simulated optimal shapes for plates P1-S-Cylinder, P2-S-Saddle and P3-S-Saddle. (a) Simulated cylindrical shape with a deflection $\mathrm{D}_{\text {sim }}$ of $1.69 \mathrm{~mm}$. (b) Initial deflection $\mathrm{D}_{i n i}$ of $0.23 \mathrm{~mm}$ in the as-received plate before peening. (c) Experimental scan of peened shape $z(x, y)_{\exp }$ after shot peening. (d) Difference between the peened shape and the simulated cylindrical shape with a maximum $\mathrm{D}_{e-s}$ of $\pm 0.24 \mathrm{~mm}$. (e) Simulated saddle shape with a deflection $\mathrm{D}_{\text {sim }}$ of $1.83 \mathrm{~mm}$. (f) Initial deflection $\mathrm{D}_{i n i}$ of $0.24 \mathrm{~mm}$ in the as-received plate before peening. (g) Scan of the peened shape $z(x, y)_{\text {exp }}$ after shot peening. (h) Difference between the peened shape and the simulated saddle shape with a maximum $\mathrm{D}_{e-s}$ of $\pm 0.22 \mathrm{~mm}$. (i) Simulated saddle shape with a deflection $\mathrm{D}_{\text {sim }}$ of $1.83 \mathrm{~mm}$. (j) Initial deflection $\mathrm{D}_{\text {ini }}$ of $1.07 \mathrm{~mm}$ in the as-received plate before peening. (k) Scan of the peened shape $z(x, y)_{\exp }$ after shot peening. (l) Difference between the peened shape and the simulated saddle shape with a maximum $\mathrm{D}_{e-s}$ of $\pm 1.0 \mathrm{~mm}$. 
the simulated optimal shape $\mathrm{D}_{e-s}$ was $\pm 0.24 \mathrm{~mm}$. Plate P2-S-Saddle had an initial deflection $\mathrm{D}_{i n i}$ of $0.24 \mathrm{~mm}$ and the maximum difference $\mathrm{D}_{e-s}$ was \pm $0.22 \mathrm{~mm}$. Plate P3-S-Saddle had an initial deflection $\mathrm{D}_{i n i}$ of $1.07 \mathrm{~mm}$ and the maximum difference $\mathrm{D}_{e-s}$ was $\pm 1.0 \mathrm{~mm}$. These observations suggest that the initial deflections have a significant impact on the final peen forming process outcome. This situation is further emphasized by the fact that plates P2-SSaddle and P3-S-Saddle, which had different initial geometries, were peened with the same patterns and were supposed to achieve the same saddle shapes, presented different differences in their final geometries.

\subsection{Plates having dimensions of $762 \times 762 \times 4.9 \mathrm{~mm}$}

The results presented so far were limited to small deflections and linear finite element models. We now turn to experiments conducted on $762 \times 762 \times 4.9 \mathrm{~mm}$ plates that exhibited sufficiently large deformations so that geometrically nonlinear effects had to be accounted for. For the plates P4-L-Saddle and P5-L-Saddle, the target deflection $\mathrm{D}_{\text {tar }}$ was $14.52 \mathrm{~mm}$ and was about 3 times their thickness $(4.9 \mathrm{~mm})$, which corresponds to the beginning of the geometrically nonlinear regime. Therefore, the optimization procedure was extended to large-deflection scenarios by replacing the linear plate model with one that includes geometric nonlinear effects, as introduced in Section 2 .

Figure 8 shows the computed peening patterns for plates P4-L-Saddle and P5-L-Saddle, with the same peening treatment, the same target saddle shape, but using linear and nonlinear plate models, respectively. Figure 8(b) presents the peening pattern computed from the linear model, which was the same as that of Figure 6(e) to achieve the saddle shape for plates P2-S-Saddle and P3S-Saddle. Figure 8(e) shows the peening pattern computed from the nonlinear model, where the optimal pattern features an outer ring peened on both faces surrounding an unpeened region. Note the similarity between these patterns and the experiments reported by Pezzulla et al. (2015) in the context of soft matter physics, where the assembly of two non-conforming concentric polymer rings causes the system to pop out of plane and assume a saddle shape. Because 


\section{P4-L-Saddle}

P5-L-Saddle

(a)

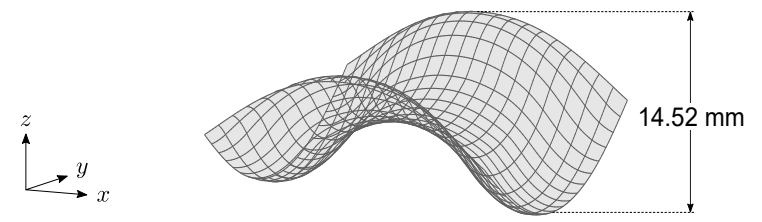

(b)

Pattern top face

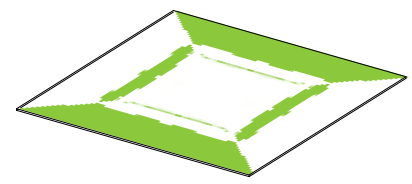

Pattern btm. face

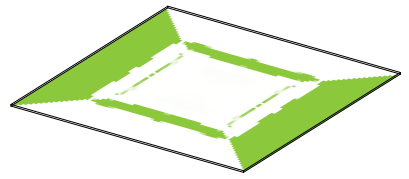

(c)
$\begin{aligned} & \text { Deformed } \\ & \text { shapes }\end{aligned}$

shapes
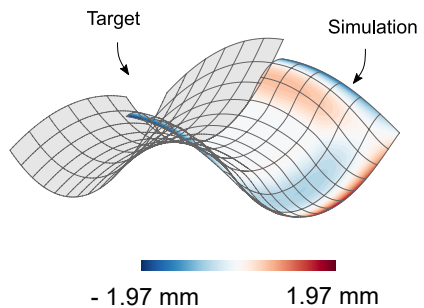

(d)

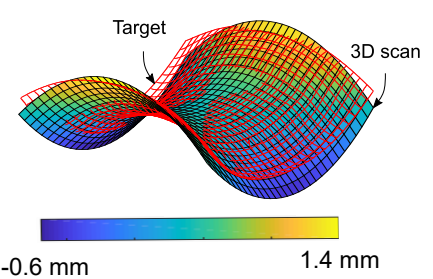

(e)
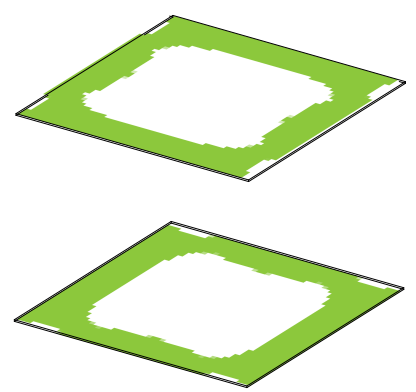

(f)

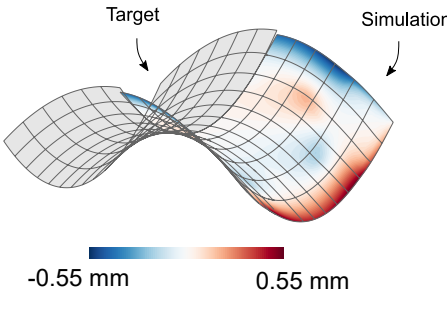

(g)

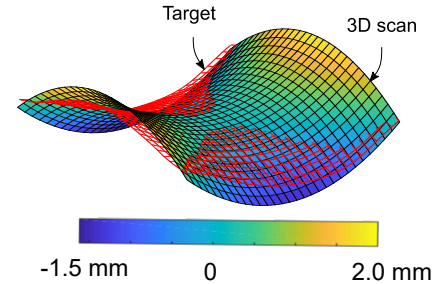

Figure 8: Optimal peening patterns computed with the linear and nonlinear optimization codes for plates P4-L-Saddle and P5-L-Saddle. (a) Target saddle shape with a target deflection $\mathrm{D}_{\text {tar }}$ of $14.52 \mathrm{~mm}$ for both plates. (b) Optimized peening patterns on the top and bottom faces of plate P5-L-Saddle to achieve saddle shape with the linear code. (c) Difference between the simulated optimal shape and the target shape with a maximum $\mathrm{D}_{s-t}$ of $\pm 1.97 \mathrm{~mm}$ using the linear code. (d) Difference between the peened shape and the target with a maximum $\mathrm{D}_{e-t}$ of $1.4 \mathrm{~mm}$. (e) Optimized peening patterns on the top and bottom faces of plate P6-L-Saddle to achieve saddle shape with the nonlinear code. (f) Difference between the simulated shape and the target shape with a maximum $\mathrm{D}_{s-t}$ of $\pm 0.55 \mathrm{~mm}$ using the nonlinear code. (g) Difference between the peened shape and the target shape with a maximum $\mathrm{D}_{e-t}$ of $2.0 \mathrm{~mm}$. 
the linear code cannot capture this instability, it returns a markedly different pattern as in Figure 8(b). However, since out-of-plane deflections are moderate, the deformed shape obtained with this pattern stays close to the target shape, except near the rim of the plate where it causes the edges to curl.

The maximum differences on $z$-displacements between the simulated optimal shape and the target shape $\mathrm{D}_{s-t}$ were $\pm 1.97 \mathrm{~mm}$ for plate P4-L-Saddle with the linear pattern and were $\pm 0.55 \mathrm{~mm}$ for plate P5-L-Saddle with the nonlinear pattern. The maximum differences between the peened shape and target shape $\mathrm{D}_{e-t}$ were $1.4 \mathrm{~mm}$ for plate P4-L-Saddle and were $2.0 \mathrm{~mm}$ for plate P5-L-Saddle. Part of this larger difference for plate P5-L-saddle could come from its initial deflection $\mathrm{D}_{\text {ini }}$, which was not measured before the peen forming test.

\section{Discussion}

5.1. The differences between the optimization process and resulting peened geometry

Table 2 lists the maximum initial deflection $\mathrm{D}_{\text {ini }}$, the maximum relative difference between the initial shape and the target shape $\mathrm{RD}_{i-t}$, the maximum simulated deflection $\mathrm{D}_{\text {sim }}$, the maximum relative differences between the simu335 lated shape and the target shape $\mathrm{RD}_{s-t}$, the maximum peened plate deflection $\mathrm{D}_{\text {exp }}$, the maximum relative difference between the peened shape and the simulated shape $\mathrm{RD}_{e-s}$, and between the peened shape and the target shape $\mathrm{RD}_{e-t}$ for the five studied plates.

For the three plates having dimensions of $305 \mathrm{~mm} \times 305 \mathrm{~mm} \times 4.9 \mathrm{~mm}$, smaller target deflections were expected and the linear optimization code was applied and validated. The maximum relative differences between the simulated shape and target shape $\mathrm{RD}_{s-t}$ was $12.6 \%$ for plate P1-S-Cylinder, and was 15.9\% for plates P2-S-Saddle and P3-S-Saddle. Experimentally formed shapes, using the predicted peening patterns, presented consistent final shapes with a relative difference $\mathrm{RD}_{e-t}$ of $9.2 \%$ for plate P1-S-Cylinder, $10.6 \%$ for plate P2S-Saddle, and $25.5 \%$ for plate P3-S-Saddle. However, the larger difference in 
Table 2: Initial deflections $\mathrm{D}_{i n i}$, relative differences between the initial shapes and the target shapes $\mathrm{RD}_{i-t}$, simulated deflections $\mathrm{D}_{\text {sim }}$, relative differences between the simulated shape and the target shape $\mathrm{RD}_{s-t}$, peened plate deflections $\mathrm{D}_{\text {exp }}$, relative difference between the peen shape and the simulated shape $\mathrm{RD}_{e-s}$, and relative difference between the peened shape and the target shape $\mathrm{RD}_{e-t}$ for the five plates considered in this study.

\begin{tabular}{llllllll}
\hline Plate name & $\begin{array}{l}\mathrm{D}_{\text {ini }} \\
(\mathrm{mm})\end{array}$ & $\begin{array}{l}\mathrm{RD}_{i-t} \\
(\%)\end{array}$ & $\begin{array}{l}\mathrm{D}_{\text {sim }} \\
(\mathrm{mm})\end{array}$ & $\begin{array}{l}\mathrm{RD}_{s-t} \\
(\%)\end{array}$ & $\begin{array}{l}\mathrm{D}_{\text {exp }} \\
(\mathrm{mm})\end{array}$ & $\begin{array}{l}\mathrm{RD}_{e-s} \\
(\%)\end{array}$ & $\begin{array}{l}\mathrm{RD}_{e-t} \\
(\%)\end{array}$ \\
\hline P1-S-Cylinder & 0.23 & 6.6 & 1.69 & 12.6 & 2.06 & 13.8 & 9.2 \\
P2-S-Saddle & 0.24 & 4.8 & 1.83 & 15.9 & 1.98 & 8.8 & 10.6 \\
P3-S-Saddle & 1.07 & 21.3 & 1.83 & 15.9 & 1.23 & 39.8 & 25.5 \\
P4-L-Saddle & $\mathrm{n} / \mathrm{a}$ & $\mathrm{n} / \mathrm{a}$ & 10.88 & 13.6 & 9.98 & 3.1 & 9.6 \\
P5-L-Saddle & $\mathrm{n} / \mathrm{a}$ & $\mathrm{n} / \mathrm{a}$ & 13.65 & 3.8 & 10.62 & 10.5 & 13.8 \\
\hline
\end{tabular}

plate P3-S-Saddle could be related to its larger initial relative deflection $\mathrm{RD}_{i-t}$ of $21.3 \%$.

For the two larger plates with dimensions of $762 \mathrm{~mm} \times 762 \mathrm{~mm} \times 4.9 \mathrm{~mm}$, a nonlinear model was required and applied. Using a nonlinear code led to patterns that significantly differed from those obtained from a linear code. While deflection measurements on peened plates showed that the patterns calculated with both linear and nonlinear codes produced similar final shapes, with maximum relative differences $\mathrm{RD}_{e-t}$ of $10.5 \%$ for plate P4-L-Saddle and of $13.8 \%$ for plate P5-L-Saddle. This is mainly due to the fact that the target deflection was still considered as moderate deformations for the plates.

The discrepancy between the target shape resulting from the optimization procedures and that experimentally measured could be explained by two main reasons:

1. The initial deformations of the aluminium plates present before peening were neglected in the optimization procedure. The plates were assumed to be initially perfectly flat in the optimization procedure. Figure 7 shows similarities between the as-received initial deflection $\mathrm{D}_{i n i}$ and the maximum difference between the experimentally measured shape and simulated saddle shape $\mathrm{D}_{e-s}$.

2. In the models presented in this paper, it is assumed that the amplitude of the plastic deformations induced by the treatment is independent of the 
position on the plate. In practice, the treatment is not instantaneous. The assumption that we made was to assume that the plate was held flat during shot peening, and that all stresses are released at the end. The progressive deformation of the plates causes the amplitude of the eigenstrains to vary in the plate. However in the whole optimization procedure, a consistent eigenstrain profile was used in the simulation, which could bring certain differences.

\subsection{Automating the identification of peening patterns and process parameters}

In the optimization process introduced in this work, the peening treatment was selected beforehand and target shapes were chosen such that they could be approached with this treatment. This was done iteratively by increasing the curvatures of the target shapes until the optimal shape could not keep pace with the target. In practice, however, it is the opposite problem that needs to be solved: the target shape is a requirement and the objective is (i) to determine whether the prescribed shape can be peen formed and, when this is the case, (ii) to find a peening pattern and process parameters that yield the desired shape within acceptable tolerances. In addition, the treatment must be compatible with production requirements. The latter usually include using as few different shot types as possible, ensuring that the maximum allowed intensity and coverage are not exceeded, and maximizing the smoothness of the patterns - as intricate patterns with many details and sudden variations of intensity or coverage are harder to manufacture than smoother, simpler patterns. To achieve this, we propose the following four-step procedure:

1. Run the optimization procedure presented in the paper without any constraint apart from the upper and lower bound on $\chi$ and with a very large value for the amplitude of the expansion in the active layer. For example, use the $\left\{h_{e q}, \varepsilon_{e q}^{*}\right\}$ for the most intense treatment that the available peening equipment can deliver. If, at the end of this analysis, the optimal shape matches the target shape within tolerances, proceed to step 2. Otherwise, the target shape can likely not be peen formed. 
2. Re-run the analysis by progressively decreasing the intensity of the treatment until the difference between the optimal shape and the target shape becomes unacceptable - using the same metrics as those used to accept or reject a part to define 'acceptable'. This gives a lower bound on the intensity of the peening treatment.

3. Identify process parameters that (i) deliver an intensity within the upper bound of step 1 and the lower bound of step 2, and (ii) are compatible with production requirements. For example, only a finite number of shot type might be available, and certain combinations of intensity and shot size might be prescribed if they result in a poor surface finish.

4. Choose one treatment, re-run the analysis by progressively adding or tightening constrains to steer the optimal solution towards a peening pattern that can be easily manufactured. For example, decreasing the upper limit on the area that is allowed to be peened tends to force the algorithm towards $[0-1]$ designs that can be manufactured by masking part of surface and indiscriminately blasting the entire surface. Similar [0-1] designs can be obtained with appropriate penalty schemes, as described in Bendsøe and Sigmund (2004) and Pajot et al. (2006). When automated peening equipment is available that can continuously adjust process parameters, less severe constraints that allow for smooth transitions between peened and unpeened areas can be used, such as those listed in VanLuchene and Cramer (1996). Note that, although they can improve the manufacturability of a pattern, constraints inevitably degrade the agreement between the optimal solution and the desired shape. If, at the end of this step, the optimal shape does match the target shape within tolerances, return to step 3 and select a different treatment.

To illustrate the third step, suppose that a perfect [0-1] pattern has been 425 computed, that is, that all components of the optimal $\chi$ are either zero or equal to the same value, $\chi$. We now wish to find a treatment that induces the same axial loads and bending moments as a step of depth $h_{e q}$ and of magnitude 
$\chi \varepsilon_{e q}^{*}$. Unless fast and accurate impact models are available, this must be done experimentally. To narrow down the search, a preliminary step would be to build databases of $\left\{h_{e q}, \varepsilon_{e q}^{*}\right\}$ versus process parameters using available experimental data sets. Most companies and research teams involved with peening processes already have databases of residual stress profiles at their disposals from which it is possible to extract eigenstrain profiles using the procedures introduced in Faucheux et al. (2018).

\section{Conclusions}

In this article, we demonstrated that it is possible to procedurally generate peening patterns for given complex target shapes. In particular, we managed to form a cylindrical shape and a saddle shape from thick plates, which are usually manufactured by costly stress peen forming operation. Our strategy relied on the combination of (i) an idealization of peening induced loads as anelastic expansion (Faucheux et al. (2018)) and (ii) an optimization algorithm (Pajot et al. (2006)).

The formulation of applying eigenstrains into a finite element model proved to be efficient for the peening pattern optimization procedure. The simulated peening patterns $[0-1]$ were easily performed by just masking the unpeened surface of the plate without changing other peening parameters. The optimization procedure could predict the peening patterns to achieve cylindrical or saddle shapes with a maximum relative difference of $15.9 \%$. Experimental validations show that using the predicted peening patterns produced consistent target cylindrical or saddle shapes with a maximum relative difference of $13.8 \%$, but with an exception of a maximum relative difference of $25.5 \%$, in which case, the plate P3-S-Saddle had a large initial relative deflection of $21.3 \%$.

For the future improvement of the optimization model, the initial deflection of the plate and the progressive deformation of the plates during the peen forming process should be considered. 


\section{Declaration of Competing Interest}

The authors declare that they have no known competing financial interests or personal relationships that could have appeared to influence the work reported in this paper.

\section{Acknowledgment}

The authors gratefully acknowledge financial support from the Canada Research Chairs program, and from Discovery Grants program by Natural Sciences and Engineering Research Council of Canada (NSERC). The authors would also like to thank Dr. P. Faucheux for original ideas behind this work and the experimental results.

\section{References}

AMS Standard 2430, 2015. Shot Peening, Automatic. Standard. SAE International.

ASTM standard E837-08, 2008. Standard Test Method for Determining Residual Stresses by the Hole-Drilling Strain-Gage Method. Standard. ASTM International. West Conshohocken, PA.

Batoz, J.L., Dhatt, G., 1992. Modélisation des structures par éléments finis. Volume 3, coques. Presses de l'Université Laval, Sainte-Foy.

Bendsøe, M.P., Sigmund, O., 2004. Topology optimization. Springer Berlin Heidelberg, Berlin, Heidelberg.

Bendsoe, M.P., Sigmund, O., 2013. Topology optimization: theory, methods, and applications. Springer Science \& Business Media.

Caselli, F., Bisegna, P., 2013. Polar decomposition based corotational framework for triangular shell elements with distributed loads. International Journal for Numerical Methods in Engineering 95, 499-528. doi 10.1002/nme.4528. 
Faucheux, P.A., Gosselin, F.P., Lévesque, M., 2018. Simulating shot peen forming with eigenstrains. Journal of Materials Processing Technology 254, 135144. doi $10.1016 / j \cdot j m a t p r o t e c .2017 .11 .036$.

Hornauer, K., Kohler, W., 1990. Development of the peen forming process for spherical shaped components, in: Proceedings of the 4th International Conference on Shot Peening, ICSP, pp. 585-594.

Irschik, H., 2002. A review on static and dynamic shape control of structures

口 by piezoelectric actuation. Engineering Structures 24, 5-11. doi 10.1016/ S0141-0296(01) 00081-5

Koconis, D.B., Kollár, L.P., Springer, G.S., 1994. Shape control of composite plates and shells with embedded actuators. II. Desired shape specified. Journal of Composite Materials 28, 262-285.

Kopp, R., Schulz, J., 2002. Optimising the double-sided simultaneous shot peen forming. ICSP-8 (Garmisch-Partenkirchen, Germany), 227-233.

Luo, M., Hu, Y., Hu, L., Yao, Z., 2020. Efficient process planning of laser peen forming for complex shaping with distributed eigen-moment. Journal of Materials Processing Technology 279, 116588.

Miao, H., Demers, D., Larose, S., Perron, C., Lévesque, M., 2010. Experimental study of shot peening and stress peen forming. Journal of Materials Processing Technology 210, 2089-2102.

Pajot, J.M., Maute, K., 2006. Analytical sensitivity analysis of geometrically nonlinear structures based on the co-rotational finite element method. Finite Elements in Analysis and Design 42,900-913. doi 10.1016/j.finel.2006. 01.007 .

Pajot, J.M., Maute, K., Zhang, Y., Dunn, M.L., 2006. Design of patterned multilayer films with eigenstrains by topology optimization. International

घ Journal of Solids and Structures 43, 1832-1853. doi:10.1016/j.ijsolstr. 2005.03 .036 . 
Paradies, R., Hertwig, M., 1999. Shape control of adaptive composite reflectors.

510 ․ Composites Part B: Engineering 30, 65-78. doi 10.1016/S1359-8368(98) 00026-2.

Pezzulla, M., Shillig, S.A., Nardinocchi, P., Holmes, D.P., 2015. Morphing of geometric composites via residual swelling. Soft Matter 11, 5812-5820. doi:10.1039/C5SM00863H

Ramati, S., Levasseur, G., Kennerknecht, S., 1999. Single piece wing skin utilization via advanced peen forming technology, in: Proc. 7th Conf. Shot Peening (ICSP7), Warsaw, Poland.

SAE standard J2277, 2013. Shot Peening Coverage Determination. Standard. SAE International.

SAEJ443, 2017. Procedures for Using Standard Shot Peening test Strip. Standard. SAE International.

Siguerdidjane, W., Khameneifar, F., Gosselin, F.P., 2020. Efficient planning of peen-forming patterns via artificial neural networks. Manufacturing Letters $25,70-74$.

Thomson, G., Pridham, M., 1997. A feedback control system for laser forming. Mechatronics 7, 429-441.

VanLuchene, R.D., Cramer, E.J., 1996. Numerical modeling of a wing skin peen forming process. Journal of materials engineering and performance 5, $753-760$.

530 Wang, T., Platts, M., Levers, A., 2006. A process model for shot peen forming. Journal of Materials Processing Technology 172, 159-162.

Wang, T., Platts, M., Wu, J., 2008. The optimisation of shot peen forming processes. journal of materials processing technology 206, 78-82. 